\title{
Fatigue, Pain, Anxiety and Depression in Guillain- Barré Syndrome and Chronic Inflammatory Demyelinating Polyradiculoneuropathy
}

\author{
Ingemar S.J. Merkies ${ }^{a}$ Bernd C. Kieseier ${ }^{b}$ \\ a Department of Neurology, Maastricht University Medical Centre, Maastricht, The Netherlands; ${ }^{b}$ Department of \\ Neurology, Medical Faculty, Heinrich-Heine University, Düsseldorf, Germany
}

\section{Key Words}

Anxiety/depression · Fatigue $\cdot$ Immune-mediated polyneuropathies · Intravenous immunoglobulin · Pain

\begin{abstract}
Background: In the clinical evaluation of patients with Guillain-Barré syndrome (GBS) and chronic inflammatory demyelinating polyradiculoneuropathy (CIDP), scant attention is paid to symptoms such as fatigue, pain and anxiety/depression. We aimed at addressing seminal studies that focused on the burden of these symptoms and their impact on quality of life (QoL) in these conditions. Summary: Fatigue, pain, and anxiety/depression are increasingly being recognized in patients with GBS and CIDP, although their pathophysiological provenance remains unknown. Fatigue and pain are significant in terms of prevalence and intensity, may be a presenting symptom, and can persist for years after apparent functional recovery, suggesting residual injury. Anxiety/depression has also been examined although studies are limited. Despite their negative impact on QoL, the long-term dynamics of these symptoms in patients with GBS and particularly CIDP receiving therapy in routine clinical practice have not been systematically evaluated. Such observations formed the basis for the ongoing (GAMEDIS) studies evaluating the effect of Gamunex ${ }^{\circledR}$ on fatigue and depression in patients with CIDP, of which some preliminary data are pre-
\end{abstract}

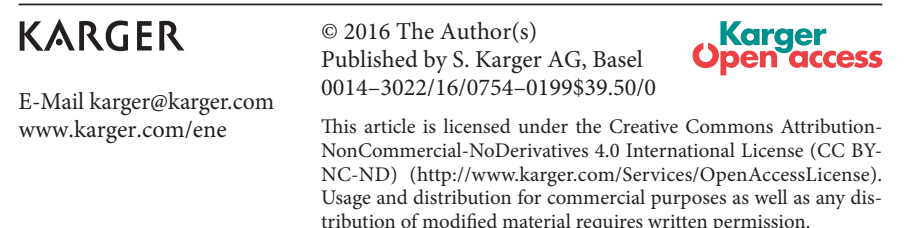

sented. Key Messages: Strength and sensory deficits are the main areas of focus in patients with GBS and CIDP, but they do not explain the total reduction in QoL, suggesting the possible role of other complaints. A more comprehensive approach to patient care demands that factors such as pain, fatigue and anxiety/depression receive greater attention. The non-interventional GAMEDIS studies are expected to provide valuable insight into the long-term effectiveness of Gamunex ${ }^{\circledR}$ in everyday practice.

(c) 2016 The Author(s)

Published by S. Karger AG, Basel

\section{Introduction}

Strength and sensory deficits dominate the clinical picture and outcome assessments in clinical trials of patients with inflammatory neuropathies, and account for $40-50 \%$ of the reduction in quality of life (QoL) expectations [1-3]. Conversely, the remaining $50-60 \%$ of the decrement in QoL expectations can be attributed to other factors. In this review, we focus on cardinal studies in patients with Guillain-Barré syndrome (GBS) and chronic inflammatory demyelinating polyradiculoneuropathy (CIDP) that examined fatigue, pain, and anxiety/depression. In addition, since the long-term dynamics of fatigue, pain, and anxiety/depression in patients with GBS and particularly CIDP receiving therapy in routine clinical 




Fig. 1. Median FSS scores in patients with immune-mediated polyneuropathies compared with HC (a); by disease (b); by gender (c). $\mathrm{HM}=$ Healthy male; $\mathrm{HF}=$ healthy female; $\mathrm{MP}=$ male patient; $\mathrm{FP}=$ female patient. Reproduced with permission from [6].

practice have not been systematically examined, we also address preliminary results of the large ongoing (GAMEDIS) studies evaluating the effect of Gamunex ${ }^{\circledR}$ ( $10 \%$ caprylate-chromatography purified immune globulin intravenous (IGIV-C); Grifols) on fatigue and depression in patients with CIDP, under conditions resembling the daily clinical setting.

\section{Fatigue in Immune-Mediated Neuropathies}

Chronic fatigue is a common symptom in neurological diseases and can be defined based on its location in the body. Peripheral fatigue is characterized by failure to sustain the force of muscle contraction [4]. Central fatigue, in contrast, is characterized by an enhanced perception of effort and limited endurance of sustained physical and mental activities [4]. Although the underlying mechanism of fatigue has yet to be elucidated, it is believed to be multifactorial, involving both peripheral and central processes [5].

The concept of examining fatigue as an outcome in immune-mediated polyneuropathies derived initially from a patient's request to have fatigue measured in addition to his strength and sensory deficits. The European Inflammatory Neuropathy Cause and Treatment (INCAT) group subsequently designed a study to determine the prevalence and severity of fatigue using the Fatigue Severity Scale (FSS) in patients with immune-mediated polyneuropathies [6]. The FSS is a 9-item self-report questionnaire that measures fatigue severity and the impact of fatigue on a person's activities and lifestyle. Responses are scored on a 7-point scale $(1=$ strongly disagree to 7 = strongly agree; score range 9-63; a higher score indicating more fatigue).
The INCAT group study involved 113 patients with previous GBS, a clinically stable CIDP or a polyneuropathy associated with monoclonal gammopathy of undetermined significance (MGUSP), and 113 age- and gendermatched healthy controls (HC). In addition to the FSS, other scales used to measure the residual effects of disease included the Medical Research Council (MRC) sumscore, functional grading scale (f-score), INCAT sensory sumscore and the 36-item short-form (SF-36) health survey [6].

The mean FSS score was significantly higher in patients than in HC (6.2 vs. 2.9; p <0.0001; fig. 1) [6]. Severe fatigue (defined as FSS $>5$ ) was present in about $80 \%$ of patients. The level of fatigue was similar in the GBS and CIDP groups, and marginally lower in the MGUSP group. In general, females reported a higher level of fatigue than males, an observation that was also evident among HC. No significant associations were found between FSS scores and values recorded for general strength, sensory deficits, GBS-disability score or symptom duration. Severe fatigue was reported in $81-86 \%$ of patients with normal strength or sensation, and the level of fatigue was similar between ambulatory and wheelchair-bound patients.

In contrast, fatigue was associated with a negative impact on patients' QoL. SF-36 health status scores were significantly lower for patients than for $\mathrm{HC}$, and the correlations between FSS scores and SF-36 scores differed by condition. In the GBS group, the strongest correlations were found in the domains of social functioning (SF; $r=$ $-0.42, \mathrm{p}=0.0001)$ and vitality $(\mathrm{r}=-0.57, \mathrm{p}<0.0001)$, while moderate, but significant, associations were also observed for mental health $(\mathrm{MH} ; \mathrm{r}=-0.30, \mathrm{p}=0.006)$ and role-emotional $(r=-0.22, p=0.04)$. Correlation with the 
socio-emotional domains of the SF-36 is thought to reflect the 'coping' element associated with a monophasic illness such as GBS. In the chronic group (i.e. CIDP/ MGUSP), the strongest correlations were found in the physical-oriented dimensions of the SF-36, specifically, general health $(\mathrm{r}=-0.65, \mathrm{p}=0.0001)$ and physical functioning $(\mathrm{PhF} ; \mathrm{r}=-0.53, \mathrm{p}=0.002)$, possibly reflecting a greater degree of uncertainty surrounding the clinical pattern of these conditions.

Subsequently, the impact of physical training on fatigue in patients with GBS and CIDP was investigated [7]. Twenty patients performed bicycle exercise training 3 times per week for 12 weeks. Although self-reported FSS scores decreased by $21 \%$ from baseline to study end (6.1 vs. $4.8, \mathrm{p}=0.001$ ), the burden of fatigue remained high. The mean FSS score of 4.8 at week 12 was still near the threshold for severe fatigue and was considerably higher than the mean score of 2.2 reported by HC.

Fatigue can also be a presenting symptom of immunemediated polyneuropathies as reported in a case series of patients who had been referred for neurological examination due to fatigue [8]. A final diagnosis of CIDP was established in all 11 patients and treatment included intravenous immunoglobulin (IVIG; $\mathrm{n}=8$ ), corticosteroid $(n=1)$ or cyclophosphamide $(n=1)$. Seven of the 10 treated patients showed significant improvement in fatigue, having a decrease in the mean FSS total score from $49.9 \pm$ 7.0 before treatment to $39.0 \pm 9.2$ after treatment. The remaining 4 patients, including the untreated patient, had stable disease.

The current clinical literature on fatigue in immunemediated disorders consists of 11 individual studies/case reports, which involved at least 2 patients with GBS, CIDP, MGUSP or multifocal motor neuropathy [9]. Across all studies, $68 \%$ of patients had severe fatigue (FSS $>5$ ), emphasizing the fact that fatigue is a serious residual symptom even in patients with apparent good functional recovery.

A review article examined the various fatigue questionnaires available for use $(>30)$, with most being ordinal-based despite the known deficiencies [8, 10-13]. To overcome these shortcomings, the Peripheral Neuropathy outcome measures Standardization (PeriNomS) study group has re-modeled the widely used FSS to a Rasch-built FSS, improving its concept and fulfilling modern clinimetric requirements in GBS and CIDP [14]. All future studies evaluating chronic conditions or conditions with chronic consequences should transform ordinal-obtained data to interval data using, for example, the Rasch method [15].

Fatigue, Pain, Anxiety/Depression in GBS and CIDP

\section{Pain in Immune-Mediated Neuropathies}

The neurological manifestations of immune-mediated disorders are variable and, in some instances, include pain $[16,17]$. Although data are limited, a few studies have examined pain and its implications in patients with GBS or CIDP.

\section{Pain in GBS}

A prospective study by a Rotterdam group was performed with the specific aim of obtaining more detailed information about pain in GBS and its clinical variants [18]. The study involved 156 patients with newly diagnosed GBS, including 18 patients with Miller Fisher syndrome (MFS). The location, type and intensity of pain were assessed with questionnaires administered at standard time points over a 1-year period.

Pain was experienced across the entire GBS spectrum, including patients with MFS, mild disease or pure motor variants of GBS. Approximately one-third of patients (36\%) reported pain as an initial symptom, occurring up to 2 weeks before the onset of weakness. Two-thirds of patients (66\%) reported pain during the acute phase of their condition (the first 3 weeks after onset) and $38 \%$ of patients reported pain during the first year. The pain type differed between the acute phase (radicular pain, meningism, painful paresthesia/dysthesia, and myalgia) and chronic phase (painful paresthesia/dysthesia, myalgia, and joint pain) of GBS although there were some areas of overlap. Among patients with pain, most $(\geq 70 \%)$ reported moderate to severe pain at each time point during follow-up (table 1). The observation that slightly more than one-third of patients reported severe pain (810 points on the 11-point (0-10) Numerical Rating Scale (NRS)) at 52 weeks after onset highlights the significant injury retained by a relevant proportion of patients with GBS.

Pain has also showed some association with intraepidermal nerve fiber density (IENFD) [19]. In the acute phase of GBS (first 3 weeks after onset), IENFD was reduced in 60 and $61.9 \%$ of patients at the distal leg and lumbar region, respectively, of which $43.7 \%$ of patients reported neuropathic pain. The IENFD at the distal leg was significantly lower in patients with pain than without pain $(\mathrm{p}<0.001)$ and correlated significantly, although moderately, with pain intensity $\left(r_{s}=-0.51 ; p=0.003\right)$, even in patients with the pure motor variant of GBS. No association was found between IENFD, clinical dysautonomia and GBS severity at nadir, as had been reported previously [20]. 
Table 1. Pain severity over time in a prospective study in patients $(n=156)$ with GBS. Reproduced with permission from [18]

\begin{tabular}{llccccc}
\hline $\begin{array}{l}\text { Pain severity, } \\
\text { n/n with pain, \% }\end{array}$ & $\begin{array}{l}\text { Maximum 2 weeks } \\
\text { before onset of weakness }\end{array}$ & Acute phase & 13 weeks & 26 weeks & 39 weeks & 52 weeks \\
\hline NRS 1-4 & $6 / 54(15)$ & $9 / 100(9)$ & $19 / 84(23)$ & $17 / 74(23)$ & $17 / 58(29)$ & $16 / 55(29)$ \\
NRS 5-7 & $25 / 54(46)$ & $36 / 100(36)$ & $30 / 84(36)$ & $28 / 74(38)$ & $22 / 58(38)$ & $20 / 55(36)$ \\
NRS 8-10 & $21 / 54(39)$ & $50 / 100(50)$ & $28 / 84(33)$ & $26 / 74(35)$ & $18 / 58(31)$ & $19 / 55(35)$ \\
Unknown & 0 & $5 / 100(5)$ & $7 / 84(8)$ & $3 / 74(4)$ & $1 / 58(2)$ & 0 \\
\hline
\end{tabular}

\section{Pain in CIDP}

Although pain is typically regarded as a secondary sign of CIDP, Boukhris et al. [21] described the characteristics of 5 patients in whom pain was the main cause of referral and the most prominent symptom. Patients presented initially with pain in the lower limbs associated with modest motor impairment (1 case), distal paresthesia (4 cases), cramps (1 case) and fatigue (2 cases). After extensive work-up including nerve biopsy, a diagnosis of CIDP was established in each case. All patients received symptomatic treatment and 4 patients received immunomodulatory treatment, which was consistently effective. Scores on the $0-10$ visual analogue scale for pain were significantly lower after vs. before treatment ( 4.1 vs. 9.0; $\mathrm{p}=0.01$ ).

In the cross-sectional survey study from the Netherlands, median pain severity as measured on the 0-10 NRS was mild and scores for patients with GBS or CIDP were similar: 2.2 vs. 2.3 points [22]. However, the proportion of patients with CIDP reporting severe pain (NRS $\geq 7$ ) was twofold higher than in patients with GBS ( 17 vs. $8 \%$; $p=0.028$ ).

In the United Kingdom, Goebel et al. [23] investigated the intensity and distribution of pain in 24 patients with CIDP. Pain intensity was categorized as mild (NRS 0-3) in $62 \%$ of patients, moderate (NRS $4-6$ ) in $26 \%$ of patients, and severe (NRS 7-10) in 13\% of patients. In this patient sample, pain typically had a distal distribution, suggestive of a length-dependent, primarily neuropathic mechanism. On the basis of their findings, the investigators concluded that 'the notion of CIDP as a painless disorder should be revisited'.

\section{Anxiety/Depression in Immune-Mediated Neuropathies}

Although chronic conditions are known to impact adversely on a person's mental state and psychiatric health [24], only a few studies have examined the psychological health status in patients with immune-mediated neuropathies.

The survey study conducted in members of the Dutch Society of Neuromuscular Disorders found that approximately 1 in $15(6.7 \%)$ of patients with GBS and 1 in 11 (9\%) patients with GBS or CIDP had depression based on Hospital Anxiety and Depression Scale scores [22].

Another group investigated psychological distress, depressive symptoms, and the health status of patients during the first year after a diagnosis of GBS [25]. At 3, 6 and 12 months post-diagnosis, patients $(n=85)$ were administered the General Health Questionnaire, Sickness Impact Profile, and Center for Epidemiologic Studies Depression Scale. Psychological distress and depressive symptoms were present at 3 months but improved notably between 3 and 6 months. While some degree of impairment of patients' psychosocial health status was still evident at 12 months, depressive symptoms had abated.

In a bicycle exercise training study in patients with GBS and CIDP, it was shown that physical training led to significant relief of anxiety and depression (table 2) [7]. Nevertheless, the impact of physical training on patients' health status was more apparent in the physical component summary (PCS) vs. the mental component summary (MCS) of the SF-36.

\section{QoL in Immune-Mediated Neuropathies}

QoL has been defined as 'the patient's reaction to the discrepancy between actual and expected achievements arising as a consequence of being ill' [26]. In general terms, QoL assessment encompasses 4 core dimensions (box 1) [27].

A study was undertaken in patients with immune-mediated polyneuropathies to determine whether QoL assessments complement conventional outcome measures [1]. The SF-36 health survey and 3 other measures (MRC sumscore, sensory sumscore, and Hughes functional 
Table 2. Anxiety/depression and SF-36 health status scores in patients with chronic inflammatory demyelinating polyradiculoneuropathy during bicycle exercise training. Adapted from [7]

\begin{tabular}{|c|c|c|c|c|}
\hline \multirow[t]{2}{*}{ Variable } & \multicolumn{3}{|c|}{ Exercise group $(n=20)$} & \multirow{2}{*}{$\begin{array}{l}\text { Healthy individuals } \\
(\mathrm{n}=10) \\
\text { baseline } \\
(\mathrm{n}=10)\end{array}$} \\
\hline & $\begin{array}{l}\text { baseline } \\
(\mathrm{n}=20)\end{array}$ & $\begin{array}{l}6 \text { weeks } \\
(\mathrm{n}=17)\end{array}$ & $\begin{array}{l}12 \text { weeks } \\
(\mathrm{n}=18)\end{array}$ & \\
\hline \multicolumn{5}{|l|}{ HADS } \\
\hline Anxiety & $1.89 \pm 0.48$ & $1.71 \pm 0.36$ & $1.59 \pm 0.30^{\dagger}$ & $1.44 \pm 0.32^{\ddagger}$ \\
\hline Depression & $1.71 \pm 0.37$ & $1.55 \pm 0.44$ & $1.39 \pm 0.28^{*}$ & $1.26 \pm 0.32^{\ddagger}$ \\
\hline \multicolumn{5}{|l|}{ SF-36 } \\
\hline PCS & 44.0 & 45.6 & $50.1^{\dagger}$ & $58.4^{\ddagger}$ \\
\hline MCS & 51.6 & 52.7 & 55.9 & 55.7 \\
\hline
\end{tabular}

${ }^{*} \mathrm{p}<0.01$ vs. baseline; ${ }^{\dagger} \mathrm{p}<0.05$ vs. baseline; ${ }^{\ddagger} \mathrm{p}<0.01 \mathrm{vs}$. baseline values of exercise group; HADS = Hospital Anxiety and Depression Scale.

\begin{tabular}{|ll|}
\hline Box 1. Quality-of-life dimensions [27] \\
\hline Physical health & Mainly disease-related and treatment-related symptoms \\
\hline Functional health & $\begin{array}{l}\text { Self-care, mobility, and physical activity level; capacity to carry out various } \\
\text { roles in relation to family and work }\end{array}$ \\
\hline $\begin{array}{l}\text { Psychosocial life } \\
\text { well-being, life satisfaction and happiness }\end{array}$ \\
\hline Social functioning & Qualitative and quantitative aspects of social contacts and interactions \\
\hline
\end{tabular}

scale) were assessed in 114 patients with clinically stable disease (GBS: 83, CIDP: 23, MGUSP: 8), and serially in 20 patients with recently diagnosed GBS $(\mathrm{n}=7)$ or CIDP $(\mathrm{n}=13)$ and changing conditions who were examined longitudinally (period: 1 year). All latter patients, except one, were treated with IVIG. Values recorded on the SF36 for both populations were compared with scores reported for the healthy Dutch community (controls).

In the cross-sectional group with stable disease, SF-36 scores were substantially lower compared with healthy Dutch control data $(\mathrm{p}<0.0001)$. The impact of disease on patients' health status was greater for the physical domains compared with the socio-emotional domains of the SF-36 (fig. 2). In the longitudinal group, treatmentrelated improvement resulted in a gradual shift of all SF36 scores toward normal values, although they were still somewhat lower than published normative data.

To date, the ICE study (IVIG for treatment of CIDP) is the largest trial to have been performed in patients with CIDP [28]. In this randomized, double-blind, response-conditional, crossover trial, 117 patients received IGIV-C or placebo every 3 weeks for up to 24 weeks in the first treatment period. Participants whose
INCAT disability score had not improved by $\geq 1$ point were switched over to the alternate treatment in a 24 week crossover period [28]. In either treatment period, participants who improved and completed treatment were eligible to be randomly reassigned to IGIV-C or placebo in a 24-week extension phase. QoL assessments were conducted using the SF-36 health survey, and results were reported separately from the efficacy and safety findings [29].

In the first 24-week period, improvements in all SF-36 domains favored IGIV-C over placebo, with values for $\mathrm{PhF}$, role-physical, SF, and $\mathrm{MH}$ reaching statistical significance $(\mathrm{p}<0.05)$; a trend was apparent in favor of IGIV-C over placebo in the domains of bodily pain, general health, vitality and role-emotional (fig. 3). Overall, treatment-related improvement with IVIG-C showed significance in the PCS score (5.7 vs. $1.3 ; \mathrm{p}=0.020$ ) but not the MCS score (3.3 vs. $0.9 ; \mathrm{p}=0.241$; fig. 3 ). Similar general trends were observed in the crossover period. During the extension phase, mean improvements in SF36 scores that had been achieved during the initial 24week treatment phase were generally increased or maintained in patients re-assigned to IGIV-C, whereas pa- 

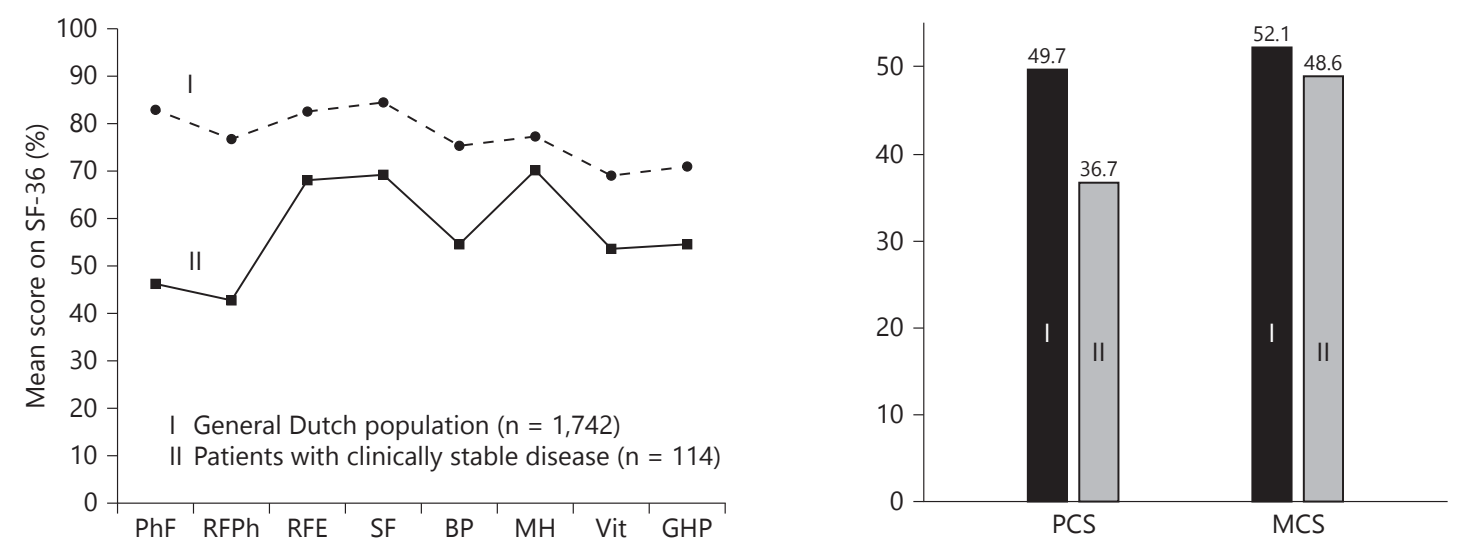

Fig. 2. SF-36 health status scores in (I) the general Dutch population and (II) the group of patients $(\mathrm{n}=114)$ with clinically stable immune-mediated polyneuropathies (GBS, chronic inflammatory demyelinating polyneuropathy, gammopathy-related polyneu- ropathy). $\mathrm{RFPh}=$ Role functioning-physical; $\mathrm{RFE}=$ role functioning-emotional; BP = body pain; Vit = vitality; GHP = general health perception. Reproduced with permission from [1].

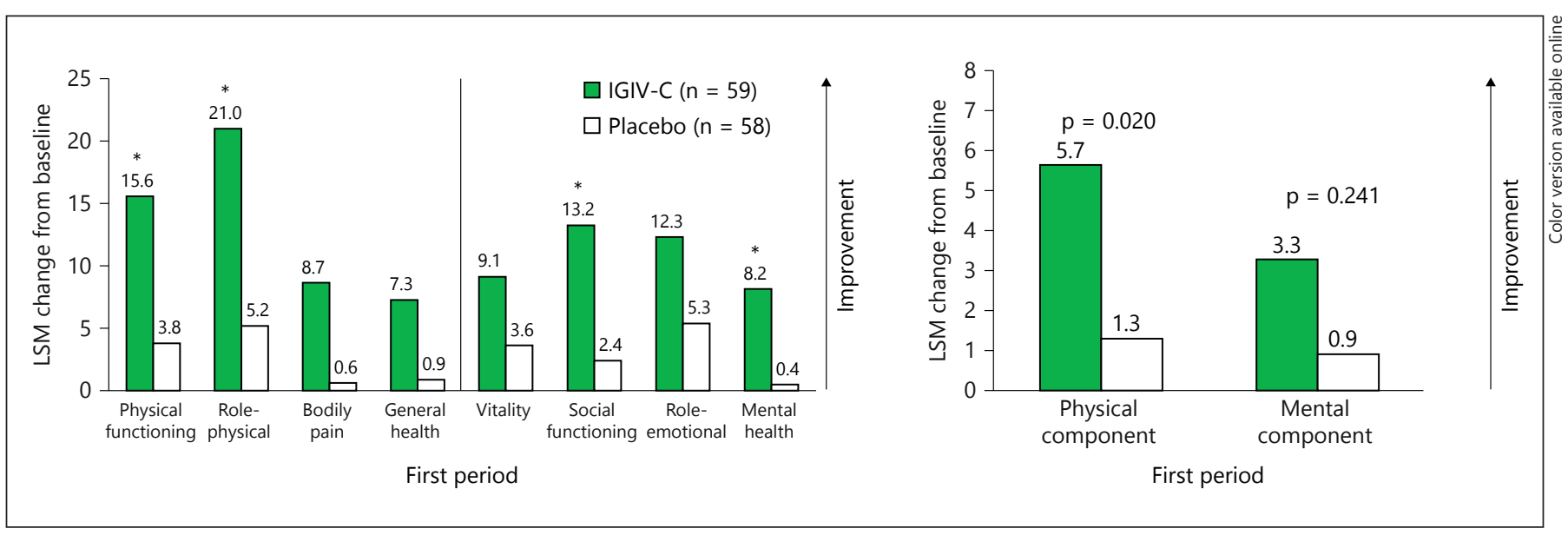

Fig. 3. QoL in patients with CIDP during 24 weeks' treatment with $10 \%$ IGIV-C (Gamunex $\left.{ }^{\circledR}\right)$ in the ICE study. Reproduced with permission from [29]. ${ }^{*} \mathrm{p}<0.05$. LSM $=$ Least squares mean.

tients re-randomized to placebo experienced worsening. SF-36 scores at baseline and after 24 and 48 weeks of treatment gradually regressed to normal values in patients who had been treated with IVIG-C for 48 weeks.

\section{Non-Interventional Study of Gamunex ${ }^{\circledR}$ in CIDP}

Clinical studies evaluating the efficacy of IVIG in patients with CIDP generally focus on muscle weakness, sensory deficit and functionality. No study to date in
CIDP has longitudinally examined the influence of IVIG on fatigue and depression dynamics in this condition. In the non-interventional GAMEDIS (GAmunex MüdigkEit DepressIon Sicherheit; Gamunex fatigue depression safety) study from Germany, changes in depression and fatigue dynamics over time were assessed in patients with CIDP treated for up to 48 weeks with IVIG. To ensure that the patient population reflected everyday practice, inclusion and exclusion criteria were kept to a minimum. Basically, all patients with a CIDP diagnosis by their treating physician, who provided signed in- 
formed consent, were eligible to participate. The sole exclusion criterion was a selective IgA deficiency. Primary outcome measures were change in depression levels on the Beck Depression Index (BDI), and change in fatigue on the FSS, from study entry through to week 48, examined initially in the traditional ordinal manner. At a later stage, most ordinal-based measures will be transformed to meet modern scientific requirements. Secondary outcomes were therapeutic benefit as assessed by IVIG therapy, general health, INCAT disability score and Hughes functional score from study entry through to week 48 . Safety was assessed by documenting all adverse events that occurred during the study.

Patients completed the BDI and FSS at study entry (visit 1 ) and every 12 weeks thereafter up to week 48 . At each 3-weekly visit, physicians assessed IVIG therapy and general health.

Thus far, 95 patients have been recruited in the GAMEDIS study. Early results indicate that fatigue remains a high burden in CIDP, whereas only a small proportion of the population were at risk for depression. IVIG has shown good safety and tolerability during longterm treatment.

Patients who completed the GAMEDIS study became eligible for enrollment in the GAMEDIS 2 study, which is extending the total observation period to 3 years. In addition to the BDI and FSS, outcome measures for patients in GAMEDIS 2 include the SF-36 health survey and Work Productivity and Activity Impairment questionnaire.

Study recruitment took place between October 2012 and December 2013 and the observation period extends from October 2012 to December 2015.

\section{Conclusions}

Fatigue, pain and anxiety/depression contribute to QoL impairment in patients with immune-mediated polyneuropathies. Since these symptoms are less tangible than strength and sensory deficit, the underlying mechanisms are not fully understood. Greater research efforts to unravel their pathophysiological mechanisms (e.g. sodium channel pathogenicity for pain?) might be expected to contribute to prognosis in immune-mediated polyneuropathies.

Although the evidence base is limited, studies that have investigated fatigue, pain and anxiety/depression in patients with GBS and CIDP have provided useful insights. Fatigue and pain can persist for many years after diagnosis, adding to the burden of disease. As fatigue and pain can also be presenting symptoms of GBS or CIDP, vigilance is required during differential diagnosis. Treatment with IVIG-C can produce relevant improvements in patients' QoL, especially for the physical components of health status.

The efficacy of IVIG-C in CIDP patients was demonstrated in the ICE study. The ongoing GAMEDIS study from Germany is the first multicenter, non-interventional trial to investigate the treatment effects of IVIG (Gamunex ${ }^{\circledR} 10 \%$ ) in CIDP patients in routine clinical practice, including assessment of depression and fatigue. Early results are in general agreement with observations from clinical studies. Final results will be reported in the near future.

\section{Acknowledgments}

Writing assistance was provided by Content Ed Net (Madrid, Spain) with funding from Grifols (Barcelona, Spain).

\section{Disclosure Statement}

I.S.J. Merkies reports grants from the GBS/CIDP Foundation International, grants from Talecris Talents program foundation, during conduct of the study; other grants as steering committee member of several studies: ICE trial, ongoing trials of Baxter, CSL Behring, LFB, and Novartis.

B.C. Kieseier has received honoraria for lecturing, travel expenses for attending meetings, and financial support for research from Bayer Health Care, Biogen Idec, Genzyme/Sanofi Aventis, Grifols, Merck Serono, Mitsubishi Europe, Novartis, Roche, Talecris, and Teva.

The authors have no other relevant affiliations or financial involvement with any organization or entity with a financial interest or financial conflict with the subject matter or materials discussed in the manuscript apart from those disclosed.

References

1 Merkies IS, Schmitz PI, van der Meché FG, Samijn JP, van Doorn PA; Inflammatory Neuropathy Cause and Treatment (INCAT) Group: Quality of life complements traditional outcome measures in immune-mediated polyneuropathies. Neurology 2002;59: 84-91.

2 Darweesh SK, Polinder S, Mulder MJ, Baena $\mathrm{CP}$, van Leeuwen N, Franco OH, Jacobs BC, van Doorn PA: Health-related quality of life in Guillain-Barré syndrome patients: a systematic review. J Peripher Nerv Syst 2014;19: 24-35.

- 3 Molenaar DS, Vermeulen M, de Visser M, de Haan R: Impact of neurologic signs and symptoms on functional status in peripheral neuropathies. Neurology 1999;52:151-156. 
$\checkmark 4$ Chaudhuri A, Behan PO: Fatigue in neurological disorders. Lancet 2004;363:978-988.

5 Garssen MP, Schillings ML, Van Doorn PA, Van Engelen BG, Zwarts MJ: Contribution of central and peripheral factors to residual fatigue in Guillain-Barré syndrome. Muscle Nerve 2007;36:93-99.

-6 Merkies IS, Schmitz PI, Samijn JP, van der Meché FG, van Doorn PA: Fatigue in immune-mediated polyneuropathies. European inflammatory neuropathy cause and treatment (INCAT) group. Neurology 1999;53:1648-1654.

7 Garssen MP, Bussmann JB, Schmitz PI, Zandbergen A, Welter TG, Merkies IS, Stam HJ, van Doorn PA: Physical training and fatigue, fitness, and quality of life in Guillain-Barré syndrome and CIDP. Neurology 2004;63: 2393-2395.

-8 Boukhris S, Magy L, Gallouedec G, Khalil M, Couratier P, Gil J, Vallat JM: Fatigue as the main presenting symptom of chronic inflammatory demyelinating polyradiculoneuropathy: a study of 11 cases. J Peripher Nerv Syst 2005; 10:329-337.

$\checkmark 9$ Merkies IS, Faber CG: Fatigue in immunemediated neuropathies. Neuromuscul Disord 2012;22(suppl 3):S203-S207.

10 Dittner AJ, Wessely SC, Brown RG: The assessment of fatigue: a practical guide for clinicians and researchers. J Psychosom Res 2004; 56:157-170.

-11 de Vries JM, Hagemans ML, Bussmann JB, van der Ploeg AT, van Doorn PA: Fatigue in neuromuscular disorders: focus on GuillainBarré syndrome and Pompe disease. Cell Mol Life Sci 2010;67:701-713.

12 Merbitz C, Morris J, Grip JC: Ordinal scales and foundations of misinference. Arch Phys Med Rehabil 1989;70:308-312.
13 Wright BD, Linacre JM: Observations are always ordinal; measurements, however, must be interval. Arch Phys Med Rehabil 1989;70: 857-860.

14 van Nes SI, Vanhoutte EK, Faber CG, Garssen M, van Doorn PA, Merkies IS; PeriNomS Study Group: Improving fatigue assessment in immune-mediated neuropathies: the modified Rasch-built fatigue severity scale. J Peripher Nerv Syst 2009;14:268-278.

15 Rasch G: Probabilistic Models for Some Intelligence and Attainment Tests. Copenhagen, Danmarks Paedagogiske Institut, 1960.

16 Said G, Krarup C: Chronic inflammatory demyelinative polyneuropathy. Handb Clin Neurol 2013;115:403-413.

17 Eldar AH, Chapman J: Guillain Barré syndrome and other immune mediated neuropathies: diagnosis and classification. Autoimmun Rev 2014;13:525-530.

18 Ruts L, Drenthen J, Jongen JL, Hop WC, Visser GH, Jacobs BC, van Doorn PA; Dutch GBS Study Group: Pain in Guillain-Barre syndrome: a long-term follow-up study. Neurology 2010;75:1439-1447.

19 Ruts L, van Doorn PA, Lombardi R, Haasdijk ED, Penza P, Tulen JH, Hempel RJ, van den Meiracker AH, Lauria G: Unmyelinated and myelinated skin nerve damage in GuillainBarré syndrome: correlation with pain and recovery. Pain 2012;153:399-409.

20 Pan CL, Tseng TJ, Lin YH, Chiang MC, Lin WM, Hsieh ST: Cutaneous innervation in Guillain-Barré syndrome: pathology and clinical correlations. Brain 2003; 126(pt 2):386397.

21 Boukhris S, Magy L, Khalil M, Sindou P, Vallat JM: Pain as the presenting symptom of chronic inflammatory demyelinating polyradiculoneuropathy (CIDP). J Neurol Sci 2007; 254:33-38.
22 Kuitwaard K, Bos-Eyssen ME, BlomkwistMarkens PH, van Doorn PA: Recurrences, vaccinations and long-term symptoms in GBS and CIDP. J Peripher Nerv Syst 2009;14: 310-315.

23 Goebel A, Lecky B, Smith LJ, Lunn MP: Pain intensity and distribution in chronic inflammatory demyelinating polyneuropathy. Muscle Nerve 2012;46:294-295.

24 Renoir T, Hasebe K, Gray L: Mind and body: how the health of the body impacts on neuropsychiatry. Front Pharmacol 2013;4:158.

25 Bernsen RA, de Jager AE, Kuijer W, van der Meché FG, Suurmeijer TP: Psychosocial dysfunction in the first year after GuillainBarré syndrome. Muscle Nerve 2010;41: 533-539.

26 Wade DT: Measurement in neurological rehabilitation. Curr Opin Neurol Neurosurg 1992;5:682-686.

27 Aaronson NK: Quality of life: what is it? How should it be measured? Oncology (Williston Park) 1988;2:69-76, 64.

28 Hughes RA, Donofrio P, Bril V, Dalakas MC, Deng C, Hanna K, Hartung HP, Latov N, Merkies IS, van Doorn PA; ICE Study Group: Intravenous immune globulin ( $10 \%$ caprylate-chromatography purified) for the treatment of chronic inflammatory demyelinating polyradiculoneuropathy (ICE study): a randomised placebo-controlled trial. Lancet Neurol 2008;7:136-144.

29 Merkies IS, Bril V, Dalakas MC, Deng C, Donofrio P, Hanna K, Hartung HP, Hughes RA, Latov N, van Doorn PA; ICE Study Group: Health-related quality-of-life improvements in CIDP with immune globulin IV 10\%: the ICE study. Neurology 2009;72: 1337-1344. 